\title{
Effect of betaine treatment on the regression of existing hepatic triglyceride accumulation and oxidative stress in rats fed on high fructose diet
}

\author{
Murat Giriş̧, Semra Doğru-Abbasoğlu², Merva Soluk-Tekkeşin³, Vakur Olgaç \\ and Müjdat Uysal ${ }^{2}$ \\ ${ }^{1}$ Aziz Sancar Experimental and Medical Research Institute, Istanbul University, Istanbul, Turkey \\ ${ }^{2}$ Department of Biochemistry, Istanbul Faculty of Medicine, Istanbul University, Istanbul, Turkey \\ ${ }^{3}$ Department of Pathology, Oncology Institute, Istanbul University, Istanbul, Turkey
}

\begin{abstract}
We investigated whether betaine has any regressive effect on existing high fructose diet (HFrD)-induced insulin resistance, dyslipidemia, inflammation as well as hepatic steatosis and oxidative stress. Rats were fed a HFrD containing $60 \%$ fructose for 8 weeks. After 8 weeks, rats were divided into two groups and fed a control diet for an additional 4-week period (regression groups). One of the regression groups received drinking water containing betaine $(1 \%$; $/ \mathrm{v})$, having antioxidant and anti-inflammatory actions. HFrD feeding caused insulin resistance, elevated triglyceride (TG) and tumor necrosis factor-alfa (TNF- $\alpha$ ) levels, alanine aminotransferase (ALT) and aspartate transaminase (AST) activities in serum. This diet increased hepatic TG, thiobarbituric acid reactive substances (TBARS) and diene conjugate (DC) levels, decreased superoxide dismutase (SOD) and glutathione peroxidase (GSH-Px) activities. Marked macro-vesicular steatosis were detected. Serum TNF- $\alpha$ and ALT, hepatic TG, TBARS and DC levels and steatosis scores decreased in regression period of HFrD-fed rats. Additionally, serum TNF- $\alpha$, hepatic TG, TBARS and DC levels significantly lower in betaine-treated regressed rats than non-treated regressed group. Our results indicate that betaine treatment may accelerate regression of HFrD-induced hepatic TG accumulation and oxidative stress in rats.
\end{abstract}

Key words: Betaine - High fructose diet - Steatosis - Oxidative stress - Liver

\section{Introduction}

Metabolic syndrome (MetS) causes high morbidity and mortality because of its cardio-metabolic complications. This syndrome is characterized by glucose intolerance, insulin resistance, dyslipidemia, hypertension, and nonalcoholic fatty liver (NAFLD) disease (Aydin et al. 2014). The increased consumption of fructose, commonly used processed food and soft drinks, is one of important factors contributing to the high prevalence of the MetS (Zhang et al. 2017). Fructose, a highly lipogenic nutrient is primar-

Correspondence to: Semra Doğru-Abbasoğlu, Istanbul University, Istanbul Medical Faculty, Department of Biochemistry, 34093, Çapa, Istanbul, Turkey

E-mail: sdabbasoglu@yahoo.com ily metabolized in liver. The exposure of the liver to high levels of fructose metabolism stimulates lipogenesis and causes triglyceride accumulation as well as glucose intolerance and insulin resistance (IR) (Zhang et al. 2017). It has been found that oxidative stress and inflammation play an important role in the pathogenesis of HFrD-induced metabolic changes (Jegatheesan and De Bandt 2017; Zhang et al. 2017). Based on these findings, several investigators have proposed the usage of antioxidant compounds to prevent HFrD-induced metabolic changes (Mohammed Salih et al. 2009; Castro et al. 2013; Giriş et al. 2014; Prabhakar et al. 2015; Sil et al. 2015).

Betaine (trimethylglycine) is a choline metabolite formed in liver by the activities of choline dehydrogenase and betaine aldehyde dehydrogenase. Also, vegetables are alimentary sources of betaine (Ueland et al. 2005). 
Betaine functions as an osmolyte and methyl group donor in methionine-homocysteine cycle. It has antioxidant and anti-inflammatory actions (Ueland et al. 2005; Day and Kempson 2016). It has been reported that betaine per se does not have a direct antioxidant activity, and that inhibition of oxidative stress by betaine is most probably related to its effect on the metabolism of sulfur-containing substances in the liver (Kim et al. 2009; Zhang et al. 2016). Indeed, betaine treatment increases S-adenosylmethionine (SAM) and glutathione (GSH) levels (Kim et al. 2009; Jung et al. 2013) and decreases homocysteine (Hcy) levels (Ueland et al. 2005). Additionally, betaine could form a protective membrane by hydrophobic interactions with phospholipids, thereby preventing the contact of free radicals with membranes (Zhang et al. 2016). Upon these effects, betaine is accepted as a hepatoprotective agent (Day and Kempson 2016). Indeed, betaine treatment is reported to have ameliorative effect in alcoholic (Balkan et al. 2004; Jung et al. 2013) and non-alcoholic fatty liver (Song et al. 2007; Kwon et al. 2009; Wang et al. 2010; Ge et al. 2016), necrotic (Balkan et al. 2005; Ahn et al. 2014; Kusku-Kiraz et al. 2018) and fibrotic (Kim et al. 2009; Bingül et al. 2016) lesions in the liver.

Some investigators were studied the effect of betaine on HFrD-induced metabolic changes in animals (Song et al. 2007; Fan et al. 2014; Ge et al. 2016). These studies have usually done to examine the preventive potential of betaine on the development of MetS. However, its regressive potential on HFrD-induced disturbances is unknown. In our study, rats were treated with/without betaine in drinking water for 4 weeks after the cessation of HFrD treatment for 8-week period. Thus we aimed to investigate the regressive potential of betaine by determining the changes in insulin resistance, lipids, hepatic oxidative stress parameters and histopathology in HFrD-fed rats.

\section{Materials and Methods}

\section{Chemicals}

Betaine anhydrous and other chemicals were purchased from Sigma-Aldrich (St Louis, Missouri, USA).

\section{Animals and diets}

Male Sprague Dawley rats weighing 190-210 g were used in the study. They were obtained from the Aziz Sancar Experimental and Medical Research Institute of Istanbul University. All the animals were housed (two or three per cage) under 12/12-h light/dark cycle. The experimental procedure used in this study met the guidelines of the Animal Care and Use Committee of the Istanbul University.
HFrD (60\% fructose diet; TD.89247) and carbohydrate control diet (TD.05075) were purchased from Harlan Teklad (Madison, WI, USA). The HFrD contained 20.7\% protein as casein, $0.3 \%$ DL-methionine, $5 \%$ fat (as lard), $60 \%$ carbohydrates as fructose, $8 \%$ cellulose, $0.004 \%$ zinc carbonate, 5\% mineral mix (170760; R-H) and 1\% vitamin mix as per weight basis (40060; Teklad). The control diet was an isocaloric modification of HFrD to replace fructose with starch $(20.7 \%$ casein, $0.3 \%$ DL-methionine, 5\% lard, $45.6 \%$ corn starch, $20 \%$ maltodextrin, $2.4 \%$ cellulose, $0.004 \%$ zinc carbonate, 5\% mineral mix (170760; R-H) and 1\% vitamin mix as per weight basis).

\section{Experimental design}

Experimental metabolic syndrome was induced by feeding the rats with $\mathrm{HFrD}$ for 8 weeks as in our previous study (Giriş et al. 2014). After 8 weeks, 8 animals selected randomly were killed (HFrD group). The remaining 16 rats were divided into 2 groups and changed to control diet for an additional 4-week period (regression groups) One of the regression groups received drinking water containing betaine $(1 \%, \mathrm{w} / \mathrm{v})$ in this period. Food and drinking water intake were controlled periodically to avoid differences between groups in the amount of consumed food and drinking water. Considering the daily water consumption, betaine intake was calculated as approximately $1 \mathrm{~g} / \mathrm{kg} /$ day. This dose was chosen according to previous studies (Balkan et al. 2005; Song et al. 2007; Kim et al. 2009; Kwon et al. 2009). In addition, 12 age-matched rats were used as a control and betaine-treated control groups ( $n=6$, each group).

At the end of this period, rats were fasted overnight and anesthetized with sodium thiopental $(50 \mathrm{mg} / \mathrm{kg}$; i.p.). Blood was collected in dry and heparinized tubes by cardiac puncture. Serum and plasma were obtained by centrifugation. Liver tissues were rapidly removed, washed in ice-cold saline and kept in ice. The liver index was calculated as liver weight/ body weight $\times 100$. The materials were stored at $-80^{\circ} \mathrm{C}$ until they were analyzed.

\section{Serum measurements}

Serum glucose, total cholesterol (TC) and triglyceride (TG) levels and alanine aminotransferase (ALT) and aspartate aminotransferase (AST) activities were determined using a Cobas Integra 800 autoanalyzer (Roche Diagnostics, Mannheim, Germany).

Insulin levels were measured using a "Rat/Mouse insulin ELISA kit" (Millipore, Billerica, MA, USA) in accordance with the manufacturer's intructions. Insulin resistance (IR) was assessed by means of the homeostasis model assessment (HOMA). The HOMA index was calculated as: fasting insulin concentration $(\mathrm{pmol} / \mathrm{l}) \times$ fasting glucose concentration 
(mmol/l)/135. High HOMA scores denote IR (low insulin sensitivity).

Plasma levels of tumor necrosis factor-alpha (TNF-a; AssayPro, UK) were measured using ELISA kits in accordance with the manufacturer's instructions.

\section{Determination of TG and TC levels in the liver}

Livers were homogenized in ice-cold $0.15 \mathrm{M} \mathrm{KCl}$ $(10 \%, w / v)$ and total lipids were extracted with a mixture of chloroform and methanol (2:1) according to the method of Folch et al. (1957) and hepatic TC and TG levels were assayed by the kits provided from Bio-Science Medical (Madrid, Spain).

Determination of thiobarbituric acid reactive substances (TBARS) and diene conjugate (DC) levels

Hepatic lipid peroxidation was evaluated by the determination of TBARS and DC levels. TBARS levels were measured by using thiobarbituric acid (Ohkawa et al. 1979). The breakdown product of 1,1,3,3-tetraethoxypropane was used as a standard. DC levels were determined spectrophotometrically in liver lipid extracts at $233 \mathrm{~nm}$ and calculated using a molar extinction coefficient of $2.52 \times 10^{4} \mathrm{M}^{-1} \cdot \mathrm{cm}^{-1}$ (Buege and Aust 1978).

Determination of antioxidant system parameters in the liver

Glutathione (GSH) levels were measured in the homogenates with 5,5-dithiobis-(2-nitrobenzoate) at $412 \mathrm{~nm}$ (Beutler et al. 1963). Superoxide dismutase (SOD) and glutathione peroxidase (GSH-Px) activities were determined in hepatic postmitochondrial fractions which were separated by sequential centrifugation. SOD activity was determined by the spectrophotometric method according to the method of Mylorie et al. (1986). GSH-Px activity was measured according to the method of Lawrence and Burk (1976) by using cumenehydroperoxide as substrate. Protein levels were assayed by bicinchoninic acid using serum albumin as standard (Smith et al. 1985).

\section{Histopathological analysis}

Liver tissues were fixed in $10 \%$ buffered formalin, embedded in paraffin, sectioned and stained with hematoxylin and eosin (H\&E) for histological studies. Steatosis was evaluated as a percentage of liver cells containing fat in the liver $(0,<5 \% ; 1,5-33 \% ; 2,34-66 \%$ and $3,>66 \%$ ) (Kleiner et al. 2005).

\section{Statistical analysis}

The results were expressed as mean $\pm \mathrm{SD}$. One-way analysis of variance (ANOVA) followed by Tukey's honestly significant difference post-hoc test was used for equal variances. Kruskal-Wallis test was performed for unequal variances. In all cases, a difference was considered significant when $p<$ 0.05 . Correlation coefficients were determined by Spearman's correlation test.

\section{Results}

Changes in final body weight, body weight gain, liver weight and liver index values

Final body weight and body weight gain per week were observed to increase in HFr group. Liver weight and liver index were also increased. Although these parameters decreased in both regression group as compared to HFr group, there was no significant difference between two regression groups (Table 1).

\section{Changes in glycemia, IR, lipid levels, hepatic marker enzy-} mes and TNF- $\alpha$ levels in serum

Feeding with HFrD significantly increased serum glucose, insulin and TG levels and HOMA values as compared to

Table 1. Effect of betaine treatment on body weights, liver weight and liver index in rats fed on high fructose (HFr) diet

\begin{tabular}{|c|c|c|c|c|c|}
\hline & $\begin{array}{l}\text { Control } \\
(n=6)\end{array}$ & $\begin{array}{l}\text { Betaine } \\
(n=6)\end{array}$ & $\begin{array}{c}\mathrm{HFr} \\
(n=8)\end{array}$ & $\begin{array}{l}\text { Regression } \\
\text { without betaine } \\
\qquad(n=8)\end{array}$ & $\begin{array}{l}\text { Regression } \\
\text { with betaine } \\
\quad(n=8)\end{array}$ \\
\hline Initial body weight (g) & $206.3 \pm 5.35$ & $208.3 \pm 3.19$ & $207.5 \pm 3.78$ & $208.1 \pm 3.72$ & $203.7 \pm 6.94$ \\
\hline Final body weight (g) & $340.5 \pm 11.2$ & $346.7 \pm 6.05$ & $364.7 \pm 10.1^{\mathrm{a}}$ & $336.5 \pm 15.5^{\mathrm{b}}$ & $326.6 \pm 24.4^{\mathrm{b}}$ \\
\hline Body weight gain (g/week) & $11.2 \pm 1.01$ & $11.5 \pm 0.69$ & $13.1 \pm 0.77^{\mathrm{a}}$ & $10.7 \pm 1.29^{b}$ & $10.2 \pm 1.52^{b}$ \\
\hline Liver weight (g) & $10.4 \pm 0.30$ & $10.3 \pm 0.27$ & $13.0 \pm 0.73^{\mathrm{a}}$ & $11.1 \pm 1.10^{\mathrm{b}}$ & $10.7 \pm 0.23^{b}$ \\
\hline Liver index $(\%)$ & $3.05 \pm 0.19$ & $2.98 \pm 0.12$ & $3.56 \pm 0.13^{\mathrm{a}}$ & $3.29 \pm 0.27^{b}$ & $3.27 \pm 0.20^{\mathrm{b}}$ \\
\hline
\end{tabular}

Data are shown as mean \pm SD. ${ }^{\mathrm{a}} p<0.05$ as compared to control group; ${ }^{\mathrm{b}} p<0.05$ as compared to HFr group. 
Table 2. Effect of betaine treatment on serum glucose, insulin, serum total cholesterol (TC), triglyceride (TG) and tumor necrosis factoralfa (TNF- $\alpha$ ) levels, homeostasis model assessment (HOMA) values, alanine aminotransferase (ALT) and aspartate aminotransferase (AST) activities in rats fed on high fructose (HFr) diet

\begin{tabular}{|c|c|c|c|c|c|}
\hline & $\begin{array}{c}\text { Control } \\
(n=6)\end{array}$ & $\begin{array}{l}\text { Betaine } \\
(n=6)\end{array}$ & $\begin{array}{c}\text { HFr } \\
(n=8)\end{array}$ & $\begin{array}{l}\text { Regression } \\
\text { without betaine } \\
\qquad(n=8)\end{array}$ & $\begin{array}{l}\text { Regression } \\
\text { with betaine } \\
\quad(n=8)\end{array}$ \\
\hline Glucose $(\mathrm{mmol} / \mathrm{l})$ & $5.68 \pm 0.39$ & $5.64 \pm 0.45$ & $8.67 \pm 1.76^{\mathrm{a}}$ & $7.99 \pm 1.26^{\mathrm{a}}$ & $7.79 \pm 1.38^{\mathrm{a}}$ \\
\hline Insulin $(\mathrm{pmol} / \mathrm{l})$ & $43.7 \pm 2.40$ & $44.1 \pm 2.07$ & $49.0 \pm 3.12^{\mathrm{a}}$ & $51.7 \pm 3.73^{\mathrm{a}}$ & $48.6 \pm 2.94^{\mathrm{a}}$ \\
\hline HOMA & $1.84 \pm 0.16$ & $1.84 \pm 0.21$ & $3.09 \pm 0.63^{\mathrm{a}}$ & $3.06 \pm 0.48^{\mathrm{a}}$ & $2.80 \pm 0.51^{\mathrm{a}}$ \\
\hline $\mathrm{TC}(\mathrm{mmol} / \mathrm{l})$ & $1.55 \pm 0.19$ & $1.52 \pm 0.16$ & $1.67 \pm 0.12$ & $1.66 \pm 0.32$ & $1.78 \pm 0.35$ \\
\hline $\mathrm{TG}(\mathrm{mmol} / \mathrm{l})$ & $0.85 \pm 0.06$ & $0.88 \pm 0.07$ & $1.65 \pm 0.17^{\mathrm{a}}$ & $1.46 \pm 0.40^{\mathrm{a}}$ & $1.44 \pm 0.35^{\mathrm{a}}$ \\
\hline $\operatorname{ALT}(\mathrm{U} / \mathrm{l})$ & $39.7 \pm 2.73$ & $40.0 \pm 2.82$ & $68.7 \pm 5.25^{\mathrm{a}}$ & $57.7 \pm 11.4^{\mathrm{a}, \mathrm{b}}$ & $51.9 \pm 5.43^{\mathrm{a}, \mathrm{b}}$ \\
\hline $\operatorname{AST}(\mathrm{U} / \mathrm{l})$ & $150.0 \pm 15.5$ & $146.7 \pm 19.4$ & $196.2 \pm 42.0^{\mathrm{a}}$ & $172.5 \pm 28.1$ & $178.9 \pm 65.7$ \\
\hline TNF- $\alpha(\mathrm{ng} / \mathrm{ml})$ & $13.3 \pm 1.55$ & $13.6 \pm 2.05$ & $26.9 \pm 4.55^{\mathrm{a}}$ & $24.0 \pm 3.40^{\mathrm{a}}$ & $19.1 \pm 3.04^{\mathrm{a}, \mathrm{b}, \mathrm{c}}$ \\
\hline
\end{tabular}

Data are shown as mean $\pm \mathrm{SD} .{ }^{\mathrm{a}} p<0.05$ as compared to control group; ${ }^{\mathrm{b}} p<0.05$ as compared to HFr group; ${ }^{\mathrm{c}} p<0.05$ as compared to regression group without betaine.

control rats, but TC levels remained unchanged. Serum ALT and AST activities and TNF- $\alpha$ levels were also higher in HFr group than control group.
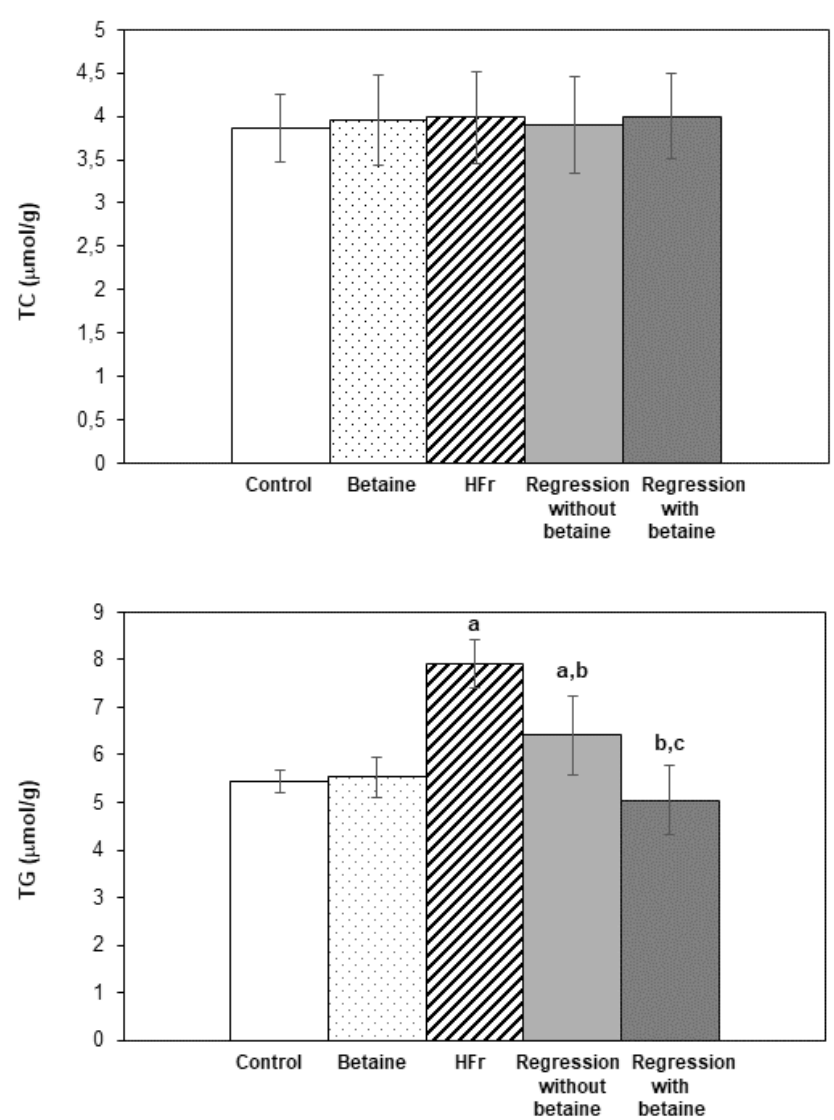

Although serum glucose, insulin, TC and TG levels, HOMA values and AST activity did not alter, ALT activity and TNF-a levels were found to decrease significantly in
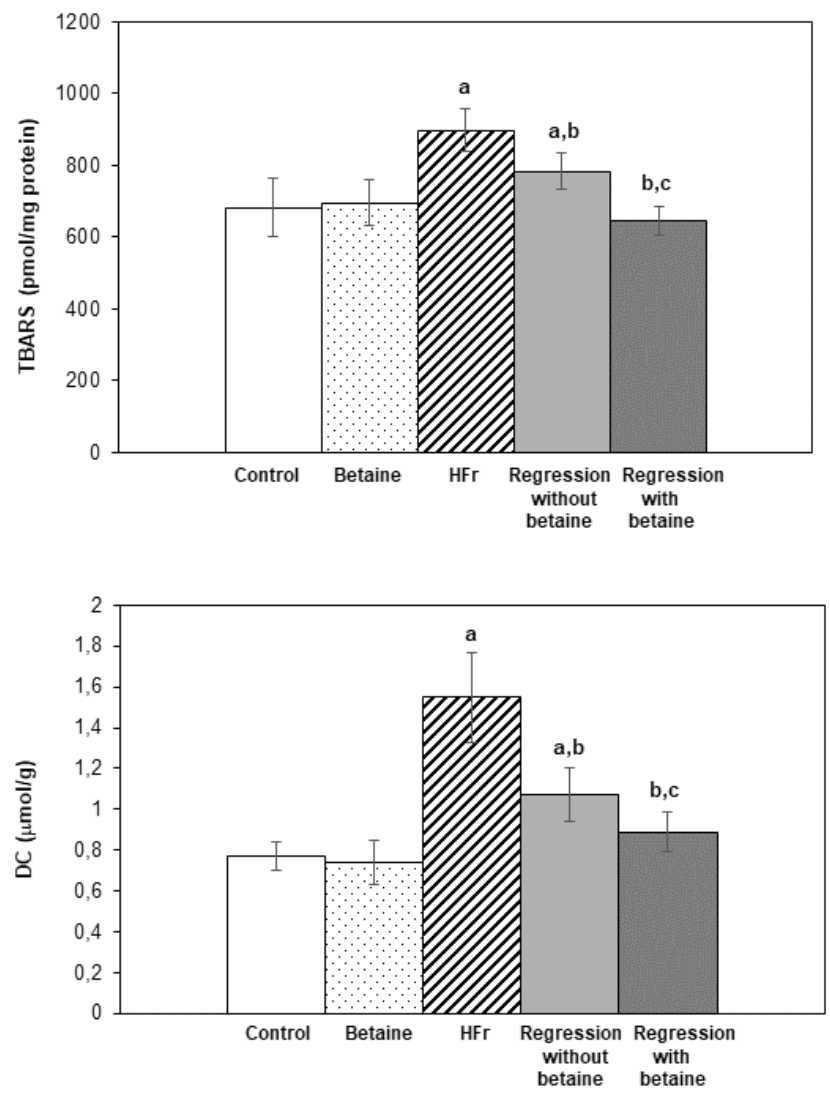

Figure 1. Effect of betaine treatment on liver total cholesterol (TC), triglyceride (TG), thiobarbituric acid reactive substances (TBARS) and diene conjugate (DC) levels in rats fed on high fructose (HFr) diet. Data are shown as mean $\pm \mathrm{SD}$. ${ }^{\mathrm{a}} p<0.05$ as compared to control group; ${ }^{\mathrm{b}} p<0.05$ as compared to HFr group; ${ }^{\mathrm{c}} p<0.05$ as compared to regression group without betaine. 
regression groups as compared to HFrD group. Only, TNF- $a$ levels were lower significantly in betaine-treated regression group than untreated regression group (Table 2).

\section{Changes in hepatic lipids, lipid peroxides and antioxidant} parameters

HFrD feeding caused significant increases in hepatic TG, TBARS and DC levels and decreases in SOD and GSH-Px activities, but GSH levels unchanged (Figs. 1 and 2). Hepatic TG, TBARS and DC levels were lower significantly in regression groups than HFrD group, but antioxidant enzyme activities did not alter. There were no changes in hepatic TC levels among groups. When regarding correlations between serum and hepatic parameters in the regression groups $(n=16)$, significant correlations were observed among serum TNF- $\alpha$ and hepatic TG $(0.577 ; p=0.019)$, TBARS $(r=0.610 ; p=0.012)$, DC $(r=0.643 ; p=0.007)$ levels in regression period. However, serum lipids, ALT and HOMA did not show any correlation with hepatic parameters (data not shown).

Hepatic TG, TBARS and DC levels were detected to be lower significantly in betaine- treated regression group than untreated regression group. Additionally, GSH levels were observed to increase in betaine-treated regression group as compared to HFrD group.

On the other hand, there were no changes in examined parameters in serum and liver of betaine-treated control rats.

\section{Histopathological findings in the liver}

Normal liver structure was seen in the control and betainetreated control rats. Marked macrovesicular steatosis was seen in the liver of rats in HFrD group. Steatosis diminished markedly in both regression groups. However, there was no significant difference between these groups (Figs. 3D,E).

Steatosis scores were detected to be significantly higher in HFr group (2.25 \pm 0.47$)$ as compared to control group (0.17 $\pm 0.41)$ These values diminished statistically in regression groups without $(0.37 \pm 0.52)$ and with $(0.53 \pm 0.52)$ betaine treatment.

\section{Discussion}

Feeding with HFrD has been largely used to produce experimental MetS in animals, since HFrD-induced metabolic disturbances have been reported to be very similar to those seen in human MetS (Aydin et al. 2014; Wong et al. 2016). This diet affects both glucose and lipid metabolism (Zhang et al. 2017) and causes hyperglycemia, IR, hypertriglyceridemia, low level of HDL-cholesterol, obesity, hypertension and fatty liver in rodents. HFrD feeding was also reported to affect the balance between oxidants and antioxidants and to increase inflammatory cytokine levels in body fluids and tissues of rodents (Mohammed Salih et al. 2009; Castro et al. 2013; Hsieh et al. 2013; Giriş et al. 2014; Prabhakar et al. 2015; Sil et al. 2015). However, several factors such as fructose
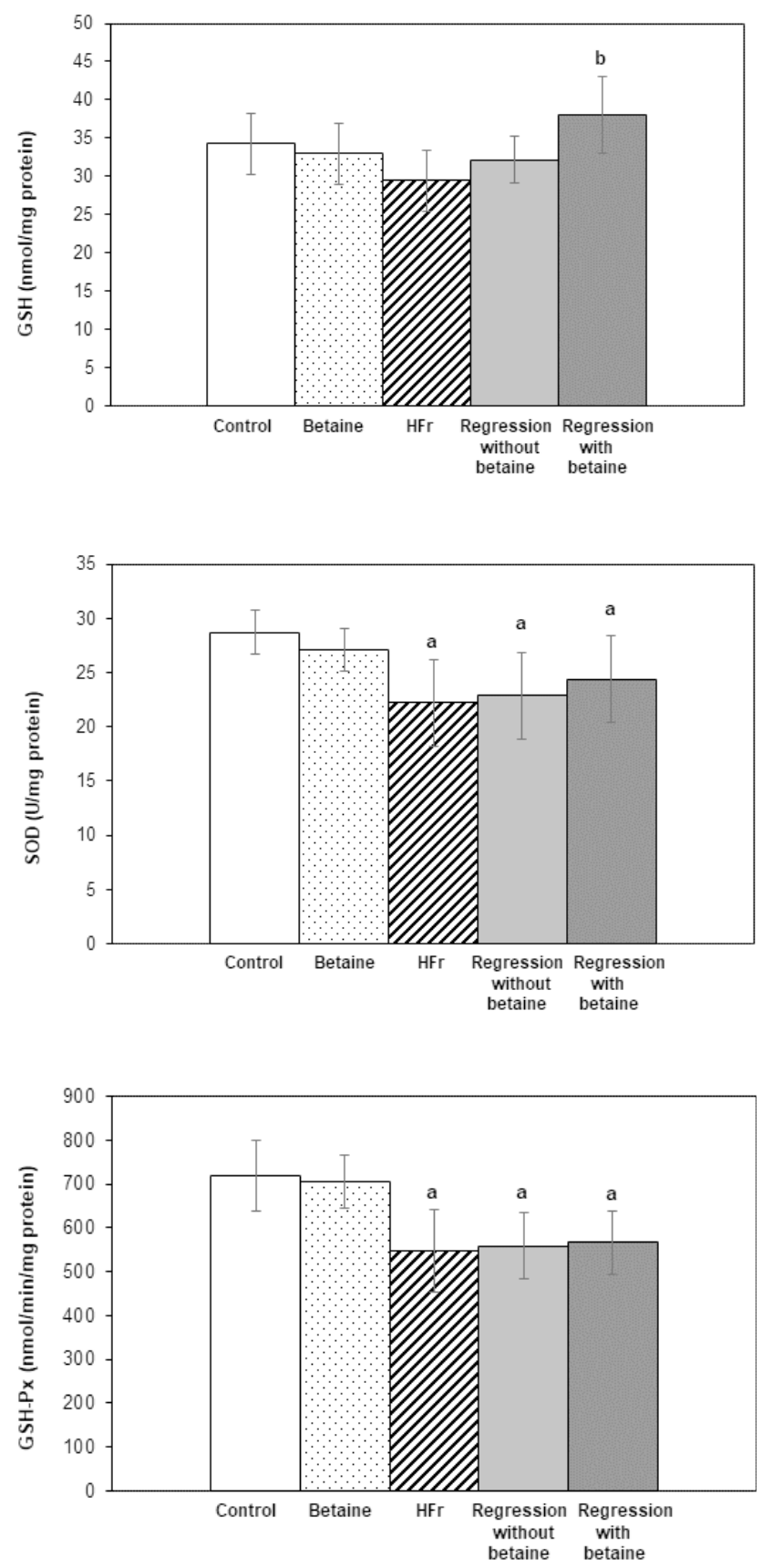

Figure 2. Effect of betaine treatment on liver glutathione (GSH) levels and superoxide dismutase (SOD) and glutathione peroxidase (GSH-Px) activities in rats fed on high fructose (HFr) diet. Data are shown as mean \pm SD. ${ }^{\mathrm{a}} p<0.05$ as compared to control group; ${ }^{\mathrm{b}} p<0.05$ as compared to HFr group. 
content of the diet, the presence of high fat component in HFrD, application time, susceptibility of tissues to oxidative damage and animal species may influence HFrD-induced changes in animals (Aydın et al. 2014; Wong et al. 2016).

Several antioxidant compounds are found to be effective in preventing a majority of the changes induced by HFrD (Mohammed Salih et al. 2009; Castro et al. 2013; Giriş et al. 2014; Prabhakar et al. 2015; Sil et al. 2015). Betaine treatment was shown to decrease IR, high levels of serum triglyceride and cholesterol and inflammation and hepatic steatosis in animals fed on HFrD (Song et al. 2007; Fan et al. 2014; Ge et al. 2016). Similar results have obtained in betaine-treated rodents fed on high fat diet, another dietary approach used to induce MetS syndrome (Kwon et al. 2009; Kathirvel et al. 2010; Wang et al. 2010; Xu et al. 2015).

Several mechanisms were reported to be effective in alleviation of IR and hepatic steatosis due to betaine treatment. These effects were attributed to normalization of downstream pathway in insulin signaling, gluconeogenesis and glycogen synthesis (Kathirvel et al. 2010), activation of hepatic AMP-
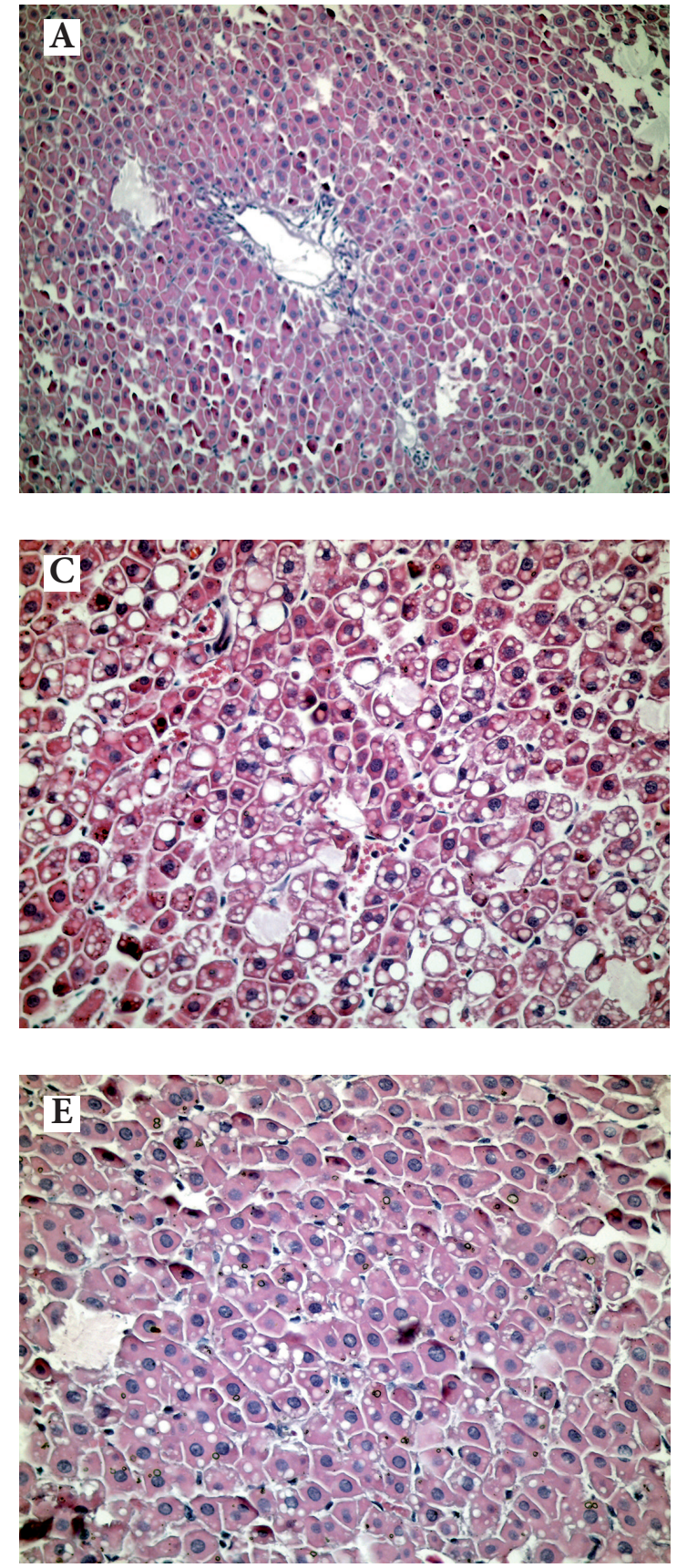
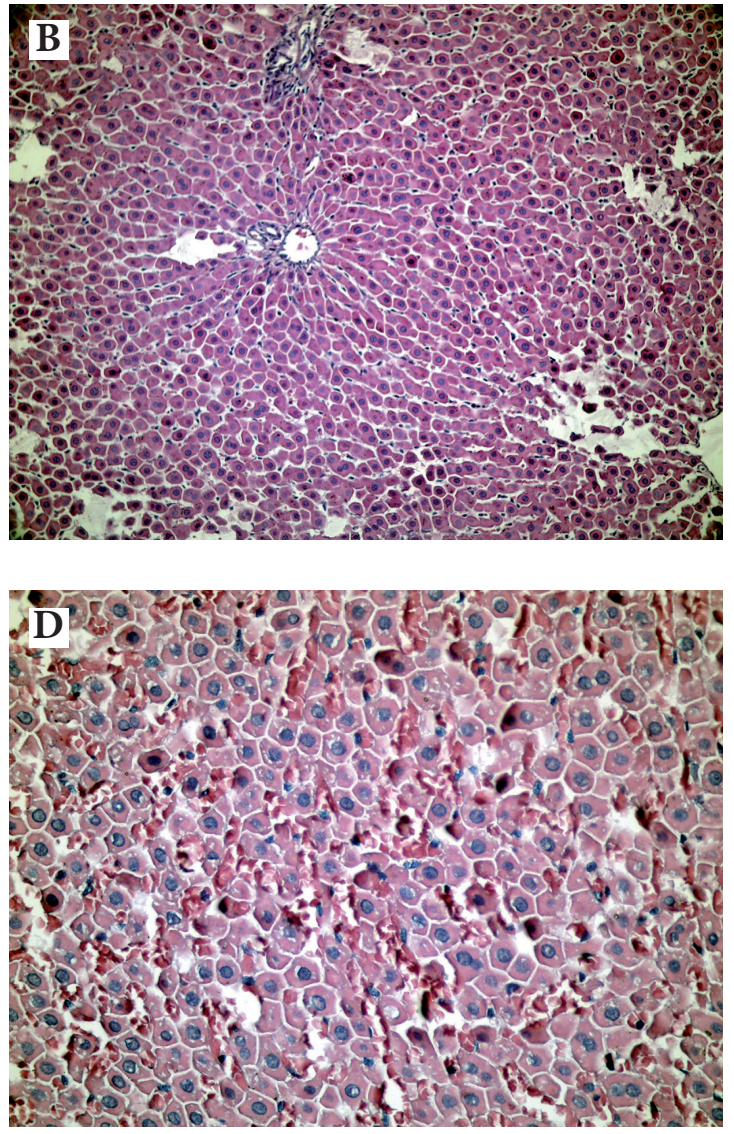

Figure 3. Effect of betaine treatment in the liver histopathology of rats fed on high fructose diet (HFrD; H\&E). A., B. Normal histologic features in control and betaine treated control groups $(\times 200)$. C. Prominent macrovesicular steatosis in HFrD group $(\times 400)$. D., E. Decreased steatosis and marked regeneration in regression groups without $(\mathrm{D}, \times 400)$ and with $(\mathrm{E}, \times 400)$ betaine treatment. 
activated protein kinase and attenuation lipogenic capability (Song et al. 2007), enhancement of fatty acid oxidation and lipid export (Xu et al. 2015). Inhibition of inflammatory factors and attenuation of endoplasmic reticulum stress (Wang et al. 2010; Ge et al. 2016), reduction of lipid peroxidation (Balkan et al. 2004) and fortification of antioxidant defence via normalization of sulfur amino acid metabolism (Kwon et al. 2009; Jung et al. 2013) have also been suggested to explain its ameliorative effect on steatosis.

In our study, we evaluated HFrD-induced metabolic disturbances such as IR, serum lipids, hepatic steatosis and oxidative stress in regression period. Final body and liver weights and liver index were observed to decrease. IR and serum lipids remained unchanged, but ALT activity and TNF- $\alpha$ levels decreased. Significant decreases in hepatic TG and lipid peroxide levels and an amelioration in histopathological steatosis scores were also seen in this period. In addition, significant correlations were observed among serum TNF- $\alpha$ and hepatic TG, TBARS and DC levels. However, serum lipids, ALT and HOMA did not show any correlation with hepatic parameters. These results indicate a parallel decrease in inflammation, hepatic steatosis and lipid peroxidation in regression period.

The main goal of this study was to investigate whether betaine treatment accelerate the regression of HFrD-induced pathological changes. There were no changes in examined parameters in serum and liver of betaine-treated control rats, as previously reported (Balkan et al. 2004; Kim et al. 2005, 2009; Bingül et al. 2016; Kusku-Kiraz et al. 2018), However, in HFrD feeding rats, decreases in serum TNF- $\alpha$ levels as well as hepatic TG, TBARS and DC levels were observed to be more pronounced in betaine-treated regression group than in untreated regression group. Hepatic GSH levels of rats in betainetreated regression group were also detected to increase, but SOD and GSH-Px activities remained unchanged. Indeed, we also demonstrated that betaine treatment decreased lipid peroxidation and increased GSH levels without any change in SOD and GSH-Px activities in the liver due to ethanol(Balkan et al. 2004) and high-fat plus carbon tetrachloridetreated animals (Bingül et al. 2016). Additionally, Zhang et al. (2016) have recently reported that betaine decreased lipid peroxides, but it did not alter activities of antioxidant enzymes (SOD, GSH-Px, catalase) in 2,2'-azobis (2-amidinopropane) hydrochloride (AAPH)-oxidised erythrocytes. These authors have also found that betaine did not affect gene expression of antioxidant enzymes (SOD, GSH-Px, catalase), whereas it upregulated endogenous nonenzymatic antioxidants such as SAM and GSH in HepG2 cells induced by AAPH. Similar increases in hepatic SAM and GSH levels were also detected in some oxidative stress-induced pathologies of the liver (Balkan et al. 2004; Kwon et al. 2009; Jung et al. 2013).

In conclusion, our results indicate that betaine treatment may accelerate the regression of HFrD-induced hepatic TG accumulation and oxidative stress in rats due to its multifunctional properties such as anti-oxidant, anti-inflammatory and anti-steatotic actions.

Acknowledgement. The present work was supported by the Istanbul University Scientific Research Projects (Project No: 6727 and BEK-2017-24919).

\section{References}

Ahn M, Park JS, Chae S, Kim S, Moon C, Hyun JW, Shin T (2014): Hepatoprotective effects of Lycium Chinense Miller fruit and its constituent in CCl4-induced hepatic damage in rats. Acta Histochem. 116, 1104-1112

https://doi.org/10.1016/j.acthis.2014.05.004

Aydın S, Aksoy A, Aydın S, Kalaycı M, Yılmaz M, Kuloglu T, Citil C, Catak Z (2014): Today's and yesterday's of pathophysiology: Biochemistry of metabolic syndrome and animal models. Nutrition 30, 1-9 https://doi.org/10.1016/j.nut.2013.05.013

Balkan J, Öztezcan S, Küçük M, Çevikbaş U, Koçak-Toker N, Uysal M (2004): The effect of betaine treatment on triglyceride levels and oxidative stress in the liver of ethanol-treated guinea pigs. Exp. Toxicol. Pathol. 55, 505-509 https://doi.org/10.1078/0940-2993-00347

Balkan J, Parıldar FH, Doğru-Abbasoğlu S, Aykaç-Toker G, Uysal M (2005): The effect of taurine or betaine pretreatment on hepatotoxicity and prooxidant status induced by lipopolysaccharide treatment in the liver of rats. Eur. J. Gastroenterol. Hepatol. 17, 917-921 https://doi.org/10.1097/00042737-200509000-00006

Beutler E, Duron O, Kelly BM (1963): Improved method for the determination of blood glutathione. J. Lab. Clin. Med. 61, 882-888

Bingül İ, Aydın AF, Başaran-Küçükgergin C, Doğan-Ekici I, Çoban J, Doğru-Abbasoğlu S, Uysal M (2016): High-fat diet plus carbon tetrachloride-induced liver fibrosis is alleviated by betaine treatment in rats. Int. Immunopharmacol. 39, 199-207 https://doi.org/10.1016/j.intimp.2016.07.028

Buege JA, Aust JD (1978): Microsomal lipid peroxidation. Methods Enzymol. 52, 302-310

Castro MC, Massa ML, Schinella G, Gagliardino JJ, Francini F (2013): Lipoic acid prevents liver metabolic changes induced by administration of a fructose-rich diet. Biochim. Biophys. Acta 1830, 2226-2232 https://doi.org/ 10.1016/j.bbagen.2012.10.010

Day CR, Kempson SA (2016): Betaine chemistry, roles, and potential use in liver disease. Biochim. Biophys. Acta 1860, 1098-1106 https://doi.org/ 10.1016/j.bbagen.2016.02.001

Fan CY, Wang MX, Ge CX, Wang X, Li JM, Kong LD (2014): Betaine supplementation protects against high-fructose-induced renal injury in rats. J. Nutr. Biochem. 25, 353-362 https://doi.org/10.1016/j.jnutbio.2013.11.010

Folch J, Lees M, Stanley GHS (1957): A simple method for the isolation and purification of total lipids from animal tissues. J. Biol. Chem. 226, 497-509 
Ge CX, Yu R,. Xu MX, Li PQ, Fan CY, Li JM, Kong LD (2016): Betaine prevented fructose-induced NAFLD by regulating LXRa/PPAR a pathway and alleviating ER stress in rats. Eur. J. Pharmacol. 770, 154-164 https://doi.org/ 10.1016/j.ejphar.2015.11.043

Giriş M, Doğru-Abbasoğlu S, Kumral A, Olgaç V, Koçak-Toker N, Uysal M (2014): Effect of carnosine alone or combined with a-tocopherol on hepatic steatosis and oxidative stress in fructoseinduced insulin resistant rats. J. Physiol. Biochem. 70, 385-395 https://doi.org/10.1007/s13105-014-0314-7

Hsieh FC, Lee CL, Chai CY, Chen WT, Lu YC, Wu CS (2013): Oral administration of Lactobacillus reuteri GMNL-263 improves insulin resistance and ameliorates hepatic steatosis in high fructose-fed rats. Nutr. Metab. (Lond) 10, 35 https://doi.org/ 10.1186/1743-7075-10-35

Jegatheesan P, De Bandt JP (2017): Fructose and NAFLD: The multifaceted aspects of fructose metabolism. Nutrients $\mathbf{9 ,} 230$ https://doi.org/10.3390/nu9030230

Jung YS, Kim SJ, Kwon DY, Ahn CW, Kim YS, Choi DW, Kim YC (2013): Alleviation of alcoholic liver injury by betaine involves an enhancement of antioxidant defence via regulation of sulfur amino acid metabolism. Food Chem. Toxicol. 62, 292-298

Kathirvel E, Morgan K, Nandgiri G, Sandoval BC, Caudill MA, Bottiglieri T, French SW, Morgan TR (2010): Betaine improves nonalcoholic fatty liver and associated hepatic insulin resistance: a potential mechanism for hepatoprotection by betaine. Am. J. Physiol. Gastrointest. Liver Physiol. 299, G1068-1077 https://doi.org/10.1152/ajpgi.00249.2010

Kim SK, Kim YC (2005): Effects of betaine-supplementation on hepatic metabolism of sulfur-containing amino acids in mice. J. Hepatol. 42, 907-913 https://doi.org/10.1016/j.jhep.2005.01.017

Kim SK, Seo JM, Chae YR, Jung YS, Park JH, Kim YC (2009): Alleviation of dimethylnitrosamine-induced liver injury and fibrosis by betaine supplementation in rats. Chem. Biol. Interact. 177, 204-211 https://doi.org/10.1016/j.cbi.2008.09.021

Kleiner DE, Brunt EM, Van Natta M, Behling C, Contos MJ, Cummings OW, Ferrell LD, Liu YC, Torbenson MS, Unalp-Arida A, et al. (2005): Design and validation of a histological scoring system for nonalcoholic fatty liver disease. Hepatology 41, 1313-1321 https://doi.org/10.1002/hep.20701

Kusku-Kiraz Z, Genç S, Bekpınar S, Ünlüçerçi Y, Çevik A, Olgaç V, Gürdöl F, Uysal M (2018): Effects of betaine supplementation on nitric oxide metabolism, atherosclerotic parameters, and fatty liverv in guinea pigs fed a high cholesterol plus methionine diet. Nutrition 45, 41-48 https://doi.org/10.1016/j.nut.2017.07.005

Kwon DY, Jung YS, Kim SJ, Park HK, Park JH, Kim YC (2009): Impaired sulfur-amino acid metabolism and oxidative stress in nonalcoholic fatty liver are alleviated by betaine supplementation in rats. J. Nutr. 139, 63-68 https://doi.org/10.3945/jn.108.094771

Lawrence RA, Burk RF (1976): Glutathione peroxidase activity in selenium deficient rat liver. Biochem. Biophys. Res. Commun. 71, 952-958
Mohamed Salih S, Nallasamy P, Muniyandi P, Periyasami V, Carani Venkatraman A (2009): Genistein improves liver function and attenuates non-alcoholic fatty liver disease in a rat model of insulin resistance. J. Diabetes 1, 278-287 https://doi.org/10.1111/j.1753-0407.2009.00045.x

Mylorie AA, Collins H, Umbles C, Kyle J (1986): Erythrocyte superoxide dismutase activity and other parameters of copper status in rats ingesting lead acetate. Toxicol. Appl. Pharmacol. 82, 512-520

Ohkawa H, Ohishi N, Yagi K (1979): Assay for lipid peroxidation in animal tissues by thiobarbituric acid reaction. Anal. Biochem. 95, 351-358

Prabhakar P, Reeta KH, Maulik SK, Dinda AK, Gupta YK (2015): Protective effect of thymoquinone against high-fructose dietinduced metabolic syndrome in rats. Eur. J. Nutr. 54, 1117-1127 https://doi.org/10.1007/s00394-014-0788-7

Sil R, Ray D, Chakraborti AS (2015): Glycyrrhizin ameliorates metabolic syndrome-induced liver damage in experimental rat model. Mol. Cell Biochem. 409, 177-189 https://doi.org/10.1007/s11010-015-2523-y

Smith PK, Krohn RI, Hermanson GT, Mallia AK, Gartner FH, Provenzano MD, Fujimoto EK, Goeke NM, Olson BJ, Klenk DC (1985): Measurement of protein using bicinchoninic acid. Anal. Biochem. 150, 76-85 https://doi.org/10.1016/0003-2697(85)90442-7

Song Z, Deaciuc I, Zhou Z, Song M, Chen T, Hill D, McClain CJ (2007): Involvement of AMP-activated protein kinase in beneficial effects of betaine on high-sucrose diet-induced hepatic steatosis. Am. J. Physiol. Gastrointest. Liver Physiol. 293, G894-902 https://doi.org/10.1152/ajpgi.00133.2007

Ueland PM, Holm PI, Hustad S (2005): Betaine: a key modulator of one-carbon metabolism and homocysteine status. Clin. Chem. Lab. Med. 43, 1069-1075 https://doi.org/10.1515/CCLM.2005.187

Wang Z, Yao T, Pini M, Zhou Z, Fantuzzi G, Song Z (2010): Betaine improved adipose tissue function in mice fed a high-fat diet: a mechanism for hepatoprotective effect of betaine in nonalcoholic fatty liver disease. Am. J. Physiol. Gastrointest. Liver Physiol. 298, G634-642 https://doi.org/10.1152/ajpgi.00249.2009

Wong SK, Chin KY, Suhaimi FH, Fairus A, Ima-Nirwana S (2016): Animal models of metabolic syndrome: a review. Nutr. Metab. 13, 65 https://doi.org/ 10.1186/s12986-016-0123-9

Xu L, Huang D, Hu Q, Wu J, Wang Y, Feng J (2015): Betaine alleviates hepatic lipid accumulation via enhancing hepatic lipid export and fatty acid oxidation in rats fat with a high-fat diet. Br. J. Nutr. 113, 1835-1843 https://doi.org/ 10.1017/S0007114515001130

Zhang DM, Jiao RQ, Kong LD (2017): High dietary fructose: Direct or indirect dangerous factors disturbing tissue and organ functions. Nutrients 9, E335 https://doi.org/10.3390/nu9040335

Zhang M, Zhang H, Li H, Lai F, Li X, Tang Y, Min T, Wu H (2016): Antioxidant mechanism of betaine with out free radical scavenging ability. J. Agric. Food Chem. 64, 7921-7930 https://doi.org/10.1021/acs.jafc.6b03592

Received: June 14, 2017

Final version accepted: February 12, 2018 